Diánoia, vol. 15, no. 15, 1969

\title{
COMENTARIO
}

\section{ALGUNAS CONTRIBUCIONES EN MI TRABAJO DE INVESTIGACIÓN}

\section{Primera Parte}

En la edición de Diánoia correspondiente a 1967, publiqué un breve comentario en torno a la investigación filosófica, indicando la importancia que tiene ocuparse de elucidar un problema que, por su propia índole, difícilmente resultaría asequible a quien no desempeñe el menester inquisitivo, siendo éste uno de los motivos por los que casi nadie se preocupa de discurrir en torno a la investigación.

En el artículo que ahora empieza me propongo llevar más adelante la proyección introspectiva sobre tan importante menester y hacerla autoscópica, al plan. tear la cuestión implicada en el título, señalando algunas de las contribuciones

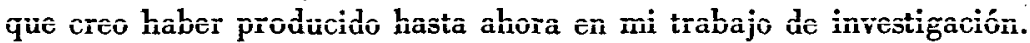

La importancia que tiene efectuar un examen personal de la obra publicada y en cierta medida de la que está en proceso de elaboración, se revela como necesario complemento a la conciencia general inquisitiva; y así debería entenderse para que cada trabajador de esta rama fuese elaborando un compendio con el decantado de sus trabajos, exponiendo lo que puede haber en ellos de contribución mayor o menor, y lo que en ello se implica, el concepto de la filosofía conforme al cual se trabaja.

La necesidad de una tal exposición se agudiza en el ambiente académico, sobre todo cuando se trata de investigadores profesionales que informan del rendimiento obtenido, para valorar el cual no existe desgraciadamente un criterio definido ni procedimientos académicos adecuados, lo cual da origen a graves actos de in. comprensión e injusticia que es necesario prevenir.

La colaboración que ahora publico en el Anuario del Instituto de Investigaciones Filosóficas, es un primer boceto autoexpositivo; contiene la apreciación general sobre algunas contribuciones aportadas en el trabajo del propio Instituto y la postura filosófica que lo orienta, lo cual podría y debería ser eventualmente una materia de diálogo académico, altamente provechoso para elucidar en cada caso el problema que reporta la valoración de la obra en cualquier investigador. Con esta inteligencia, veamos cuáles son, hasta el momento, algunas de dichas contribuciones y conforme a qué criterio se estiman como tales.

En una primera visión panorámica, el plan general de mis escritos se inicia abordando los dos grandes sectores de la investigación, o sean el sistemático y el histórico, presentados como boceto en los dos iniciales libros que publiqué. El primero, titulado Reflexiones en torno a la filosofía de la cultura traza el pros- 
pecto general de un sistema que parte del concepto predominante en la escuela axiológica del criticismo, el cual considera a la filosofía como teoría de la cultura, afocada en sus valores de realización. En tal aspecto, me parece es un intento nuevo, cuando menos en la filosofía de lengua castellana, debido al relativo aban. dono en que se tiene actualmente este criterio. El sistema que estoy tratando de construir, basado en el tratamiento analógico de las doctrinas filosóficas, admite al sistema de los valores como base. Algunos conceptos de la axiología cultural se encuentran en el capítulo cuarto de mi libro Principios de antropología. En la polémica y ensayo La esencia del valor, fueron sometidos a crítica y defensa; ahí se expone su primera formulación sistemática.

La retoma de este proyecto está contenida en el decálogo de los libros que llevan el rubro general de "Principios". Contrariamente a la opinión que pudieran motivar a quienes no se tomen la molestia de analizarlos, no constituyen simples obras de divulgación, si con ello se entiende que solamente defienden ideas ajenas; aunque me esfuerzo en producir un máximo de claridad expositiva que no daña el contenido heurístico de los mismos, lo cual buena falta hace en la literatura especializada de nuestro tiempo. Por ello, algunos de tales libros se han recomendado como textos en planteles de enseñanza superior, aunque -a excepción de un caso, el de lógica- no están sujetos a programa alguno, sino al contrario, los programas escolares se han adaptado en ocasiones al temario de estos libros.

El menos original del decálogo y el único que atiende a un programa escolar es Principios de lógica, pero aun en éste hay las suficientes aportaciones para considerarlo trabajo de investigación; en todos los demás existen contribuciones que me permitiría cuantificar grosso modo, diciendo que varían desde aproximadamente un tercera parte, como en Principios de ética o Principios de epistemologia, hasta la mayor parte del trabajo, diría un ochenta por ciento, como en Principios de estética, que no sólo es el primer libro de estética sistemática que se escribe en lengua castellana, sino también una obra de alcance mayor cuyas apreciaciones influirán en el tratadismo general de la materia.

Para decirlo a la hegeliana manera, el plan que desarrollo en los Principios comprende dos grandes vertientes, la objetiva y la subjetiva, en las cuales se recoge el temario fundamental que interesa a la filosofía en la postura axiológica que sustento. La vertiente objetiva está desarrollada a través de las disciplinas fundamentales: lógica, epistemología, estética y ética, que llevan los números 3 , 4,5 y 6 en la publicación de la Serie. La vertiente subjetiva se canaliza a través de la antropología, la psicología, la pedagogía y la axiología (ésta como vértice conjunto), la primera de las cuales está publicada con el número 2, la segunda, se encuentra en prensa con el 7 y las demás -8 y $9-$ en estado de proyecto.

El libro último de la serie llevará el número 10 y corresponderá a la historia, observada en la problemática de la filosofía de la historia o historiografía que 
preferiré llamar Historiología, en la cual se obtiene la síntesis de las vertientes. objetiva y subjetiva, producida por medio de la axiología, cuyo dinámico devenir registra la historia.

El desarrollo de la vertiente subjetiva se funda en una apreciación mía que considera a la antropología como disciplina general y metódica de las ciencias del hombre, tema éste que desarrollé en el libro Introducción a la antropología formal publicado en la colección Diánoia, en cuyas páginas efectúo un nuevo planteamiento de la problemática antropológica, al considerar que la "antropología", entendida lato sensu, no es una ciencia particular, sino la metodología formal que se refiere a todas las ciencias del hombre. La psicología, por su parte, es la ciencia medular o fundamental del ser humano, cuya aplicación a su mejoramiento da origen a la pedagogía, la cual se inspira a su vez en las finalidades estimativas representadas por la axiologia. No se trata, pues, de una recopilación de trabajos, sino de una disposición orgánica que desenvuelve el tema de lo humano a través del sistema antropología-psicología-pedagogia-axiología, que determina sus principales motivaciones y concluye con la vertiente objetiva cuya secuencia es: propedéutica-lógica-epistemología-estética-ética, vinculándose ambas dialécticamente en la historia. Obsérvese que no incluyo a la metafísica,

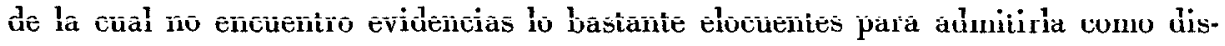
ciplina objetivamente válida. El sistema que elaboro se funda en el concepto hegeliano del ser como explicable a través de la idea, mediante el concepto universal del valor, que para mí equivale a función axiológica-dialéctica.

Tal es, en su aspecto fundamental, el sistema que estoy desarrollando y para el cual la serie "Principios" constituye apenas un boceto que pretendo desenvolver en una nueva serie de "Tratados", en la medida en que lo permitan el tiempo y las energías. Algunos temas se han ampliado en nuevas reelaboraciones, como sucede con Introducción a la antropología formal, tratamiento ulterior del concepto metódico de la antropología, que hasta ahora no se había presentado en forma sistemática. También he llevado a un amplio desenvolvimiento el temario de la estética considerada como filosofía del arte, en el Tratado de estética, obra en preparación que constará de cuatro volúmenes con una extensión total de unas 2000 cuartillas. También he dado los primeros pasos para un Tratado de psicología, que tendrá principalmente el carácter de replanteamiento y sistematización del temario respectivo.

Me permitiré exponer brevemente cuál es el carácter general de los libros que he publicado hasta ahora en la serie "Principios". Veamos desde luego el primero de ellos, o sea Principios de filosofia." Se trata de un libro elemental en cuanto básico, que en términos generales puede considerarse como un ensayo para refundar el concepto de filosofía como teoría axiológica de la cultura, aplicando el criterio del valor al sustrato universal de la cultura misma. La primera

* Editorial Patria, 1ª edición, 1958; $4^{n}$ edición, 1968. 
parte, intitulada Propedéutica, contiene los conceptos fundamentales del sistema; son ante todo, los conceptos de cultura, técnica, experiencia y método, y el que deriva de todos, o sea el concepto de la filosofia misma. Sobre lo mucho que se ha escrito en relación a tal problema, diré que en ese capítulo se encuentra el esquema comparativo de los valores, insistiendo en el distingo, pero al mismo tiempo en la complementación, de cultura y técnica; la filosofía es presentada, siguiendo la tradición dialéctica, no como una ocupación abstracta, sino como proyección concreta del conocimiento "universalista" sobre la experiencia cul. tural, entablando la simbiótica interrelación de sus conceptos fundamentales.

La segunda parte se refiere a la Historia filosófica; en ella trazo un brevísimo esquema que se sujeta a la clasificación tradicional en ocho grandes periodos, si bien considerada sincréticamente en sus sistemas como resultado de aplicar puntos de vista que, en contra de lo habitualmente creído, no se oponen sino más bien se complementan en la integración del magno sistema universal cons. tituido por la totalidad de los sistemas históricamente dados.

El capítulo de Lógica se somete al criterio del formalismo aristotélico, aunque tiende a explicar dinámicamente los sectores de la lógica formal; en este capítulo hay observaciones criticas y expositivas que de seguro no revolucionarán a la lógica, pero contribuyen a situarla en un plano dinámico, principalmente en la dialéctica de la predicación, remitido al volumen de epistemología, para corresponder a la programación del sistema.

El capítulo concerniente a la Etica se aparta por completo de los lineamientos frecuentes en la exposición de esta disciplina; presenta una ética de la autonomía basada en la conciencia y responsabilidad de la conducta. Contiene algunas contribuciones específicas, como el esquema de la praxis, la teoría del progreso moral mediante la objetivación de la conducta, el conjunto de los problemas fundamentales y el método que emplea esta disciplina, así como la clasificación y relativización de los posturas morales. Particularmente señalaré la clasificación que efectúo en el terreno de la ética heterónoma, así como la idea de una ciencia moral, fundada en el conocimiento arrojado por las diferentes ciencias particulares que se ocupan de la conducta, de acuerdo al basamento axiológico a que hice referencia con anterioridad.

El último capítulo, relativo a la Estética, recoge los conceptos fundamentales del planteamiento que establezco para esta disciplina en la obra relativa a la misma, sobre cuya originalidad y validez hablaré posteriormente con cierta extensión.

El segundo libro de esta serie es Principios de antropologia; ${ }^{*}$ contiene los materiales básicos de las disciplinas incluidas en las cuatro partes del libro: antropología, psicología, pedagogía y axiología, atendiendo a la secuencia formativa que adquieren en la trayectoria ascendente originada en el factum antropológico, donde se desplazan los hechos vitales del ser humano; asciende a la idealidad

* Editorial Patria, $1^{*}$ edición, 1962. 
axiológica que encontramos en las finalidades valorativas que se propone realizar, para lo cual pone en juego el conjunto de facultades que demuestra la psicología, encaminadas al necesario mejoramiento determinado por la pedagogía. He aquí, en pocas palabras, la perspectiva que aplico en este libro, a través de cuatro niveles doctrinarios que principian por el de mayor concreción -antropológicoy llevan al de mayor idealidad - axiológico - pasando por el psicológico y peda. gógico, que son las funciones centrales o medulares del ser humano.

El concepto que reconozco en la antropología proviene de observar el crecido número de disciplinas que desde diversos aspectos abordan el estudio del ser humano, siendo cada una de ellas una antropología específica que, sin embargo, pretende frecuentemente ostentar carácter de universalidad, o cuando menos de preferencia sobre las demás manifestaciones antropológicas. Creo que debe plantearse el problema metodológico de la antropología, cuál es la búsqueda del concepto y la idea del hombre, referido a una antropología formal. Por ello, el capítulo correspondiente a la Antropologia tiende a demostrar que cualquier conocimiento concreto en relación al hombre debe producirse en alguna de las especificaciones antropológicas, pues el concepto empírico de antropología incluye la totalidad de las ciencias del hombre que, partiendo de la experiencia, adoptan como objeto de esiudio lus feñúnienuos cãacterísticos del ser humano. De ahi las diferentes acepciones que reconozco en la antropología concreta o material: física, histórica, métrica, social, económica, etnológica, cultural y aplicada. Queda al margen la llamada "Antropología filosófica" de estilo tradicional, cuya existencia válida niego rotundamente.

La Psicología es una ciencia cuyo objeto pertenece a la esfera de lo humano; su tarea está suficientemente delimitada a la esfera de los fenómenos psíquicos, aunque tanto se ha extendido y admite cantidad tal de relaciones, que se impone una aclaración sobre los diferentes tipos de psicologia que existen. Reconozco principalmente cuatro grandes direcciones en esta ciencia: la psicología básica, analítica, biológica y social; cada una de ellas da origen a diversas subdivisiones de importancia. La tesis que presento en el primer capitulo puede enunciarse como el refrendo de la psicología básica, basamento de los conceptos psicológicos sobre los cuales se erige la superestructura específica de la psicología biológica, la psicología analítica y la psicología social.

También rechazo la posibilidad de una psicología filosófica desde el momento en que la psicología es una ciencia independiente, aunque mantiene estrechas relaciones con el saber filosófico, sobre todo en la acepción de una filosofía indesligable de los valores culturales, que en gran medida influyen a la conducta psicológica, cuya determinación preocupa esencialmente al desarrollo moderno de esta ciencia. Como corolario del esquema que presento y promuevo ampliamente en el tomo 7, Principios de psicologia, obtengo la circunscripción y relativización de las diferentes escuelas psicológicas, que por desgracia no priva como norma característica en el prospecto normal de tales disciplinas. 
En relación al problema pedagógico, efectúo una exposición fundamental en lo concerniente al proceso dinámico de la educación y su correlato con las ciencias conexas, incluyendo a la axiología, que desempeña el papel normativo y direccional señalado con anterioridad. La presentación genérica del problema tiene lugar en la fenomenología del acto educativo y el señalamiento de la influencia decisiva que sobre él ejerce la sociedad. Los problemas básicos correspondientes a la pedagogía especial se desenvuelven de manera relevante en las relaciones de pedágogía y cultura. Sobre esta perspectiva existen algunas observaciones originales para enfatizar la correlación individuo-sociedad, que no debe permitir el entronizamiento de una pedagogía radicalmente individualista o colectivista. Señalo también las condiciones básicas de la formatividad, el sentido del autodidactismo, la importancia de las fuentes educativas, la participación del Estado y la iniciativa privada, las coordenadas educacionales fácticas, la diversificación pedagógica, y sobre todo, el énfasis colocado en la función formativa de la cultura, motivos que justifican la inclusión de la pedagogía en el seno del sistema filosófico-cultural, que necesariamente conduce a la doctrina ecuménica de los valores.

Ésta se encuentra en la cuarta y última parte del libro, intitulada Axiología, que seguramente contiene la aportación más representativa del libro. Parto de la aceptación general de la cultura como realización de valores, para defender una especie de panaxiologismo, o si se prefiere, una axiología trascendental; todo género de problemas y formas del ser y el devenir son susceptibles de recibir un planteamiento axiológico. Destaco el aspecto subjetivo de los valores como sím. bolo de la vida y sintoma del carácter que los elige, teniendo en cuenta la dimensionalidad histórica y sociológica que asiste a cada tipo de elección. Ese postulado lleva a concluir que la noción del valor es la clave del progreso y denota al mismo tiempo la capacidad de vivir, de acuerdo con la fórmula que expongo: ser = valer $=$ vivir.

También el ensayo sobre las categorías del valor debe atribuirse a mi responsabilidad; presento un esquema armónico y dialéctico donde reconozco categorías téticas, heterotéticas y sintéticas, con la función que corresponde a cada una: sintesis, idealidad, relatividad, polaridad, unidad, concreción, gradación, realidad, utilidad $y$ jerarquía. Por último, el tema tan debatido en la axiología de todos los tiempos, relativo a la objetividad y subjetividad de los valores, se trata en la parte final del libro, donde llego a la conclusión de que el problema axiológico es en cierto modo una retoma del problema epistemológico, el cual debe considerarse resuelto mediante el reconocimiento de que el saber no puede aceptarse como radicalmente objetivo o subjetivo, sino ambas cosas a la vez; problema dialéctico, o sea dinámicamente correlativo, oscila entre los polos de objetividad y subjetividad, de acuerdo al sentido de la función epistémica, y en este caso axiológica, que adquiere prioridad. En última instancia, el valor, y con él todo lo que existe, es una función cuyo múltiple se integra en el sistema valorativo que contienen las posturas filosóficas, los sistemas científicos y las actitudes vitales. 
En relación a Principios de lógica debo subrayar lo que expuse anteriormente: lo considero el trabajo menos original, el único que ha tenido en cuenta la existencia de un programa escolar, se apega en sus lineamientos externos a una escuela cuyo planteamiento ha partido de la tradición escolástica y positivista, preponderante hasta ahora en nuestro medio, cuando menos en lo que a dicha disciplina respecta. Sin embargo, aun este libro contiene determinadas aportaciones que justifican su publicación, sin mengua de las profundas reformas que debe experimentar en ulteriores ediciones. El contexto relativo a la lógica formal surgió de ciertos apuntes redactados al hilo de la cátedra preparatoriana, de donde la señalada influencia del programa escolar. Empero, contiene también las suficien. tes nociones de lógica dialéctica para considerarlo como una introducción elemental a la lógica moderna, mismas que en el concepto del autor evolucionaron posteriormente hasta constituir un volumen independiente, como es Principios de epistemología. De ahí que, para evaluar el trabajo realizado en tan importante disciplina sea menester acudir a este libro, que equivale a una segunda parte de la Lógica y del cual hablaré posteriormente.

Por ahora, sujetándome al planteamiento de lógica formal, diré que principia con una expnsiriońn de las diferentés posturas que predominan en la tradición; tiendo a exponer el sincretismo funcional que manifiesta el análisis fenomenológico del conocimiento. Resulta de ahí la autonomía metodológica, aporética y sistemática, de donde la existencia de una lógica formal junto a la predicativa, mate rial, funcional y especial, desembocando en la lógica moderna, con el señalamiento dialéctico de la predicación y el problema semántico que tanto preocupa a la lógica contemporánea. Ingreso en la teoría del concepto, exponiendo sus cualidades básicas de determinación, delimitación y definición, así como el vínculo entre concepto, representación, expresión y contenido. En la clasificación de los conceptos acudo a las más reconocidas, e incluyo un nuevo análisis al excogitar las relaciones entre función lógica y función gramatical, llegando finalmente a la exposición de las categorías kantianas para enfatizar el a veces soslayado carácter dialéctico.

El capítulo relativo a las funciones conceptuadoras contiene algunos aportes como la teoría de la designación, las condiciones y formas de la definición, la teoría dinámica de la clasificación y la inordinación, que presento vinculadas a sus correlativas funciones dialécticas, cual en verdad es menester. Otro tanto con la fundamentación, que prosigo basada en los diferentes tipos de ciencias de acuerdo a su propia estructura conceptual, así como el problema dinámico de la axiomatización que es para mí el más importante en cuanto sintetiza los temas de la lógica formal y material. También considero original la forma de exponer el problema de los principios lógicos supremos mediante la confronta y crisis de los conceptos tradicionales.

El punto de partida que adopto para el análisis de la predicación - contraria-

* Editorial Patria, 1* edición, 1959; $3^{\mathrm{q}}, 1968$. 
mente a lo que se estila en lógica formal- es el juicio; considero como funciones correlativas al concepto y al razonamiento, afirmando la integridad predicativa en virtud de la dinámica epistémica que toma al concepto como elemento constitutivo del juicio y al razonamiento como estructura integrativa de judicaciones. Según este criterio, señalo diferentes tipos de juicios de acuerdo a su función cognoscitiva, para incluir después una apreciación sobre las clasificaciones más reconocidas, en primer término la tradicional y la kantiana. El capítulo del ra. zonamiento contiene la caracterización dinámica de las formas predicativas y la explicación funcional del razonamiento. Hago una revisión y en cierto modo un replanteamiento de las inferencias tradicionales, incluyendo algunos señalamientos propios, como el distingo entre inferencias necesarias, inferencias posibles e inferencias formales en el juicio. Las páginas dedicadas a la silogística y la inducción, no son más que reexposiciones de un material obligado en los libros de texto.

A diferencia del libro anterior, que contiene un minimo de aportaciones, presento los Principios de epistemología como una obra de la cual ya he dicho es el complemento integrativo y dialéctico de la lógica formal, cuya mayor parte se puede considerar trabajo de investigación sobre una temática clásica cuya secuen. cia es en cierto modo obligada. A partir del primer capítulo, que se refiere a las posturas extralógicas, procedo a la depuración de numerosas confusiones que siguen figurando en epistemología, principalmente las teorías metafísicas, psicologistas, fenomenológicas, gramaticistas y formalistas, que obedecen a confusiones metodológicas cuyo necesario deslinde establezco.

El capítulo segundo, relativo a la verdad y la realidad, parte de la epistemología crítica, eliminando algunos errores esquemáticos y metafísicos que en ella se encuentran; éste es un contexto original para fundamentar la teoria autọnoma de la experiencia mediante desarrollos que retoman ideas fragmentariamente dispuestas en los clásicos del criticismo, pero ordenadas en rigurosa unidad sistemática.

El tercer capítulo se refiere al método del conocimiento, problema insoslayable en cualquier doctrina epistemológica, pues constituye la base integrativa del saber. Considérolo como forma general del pensar, concebida mediante la unidad sincrética que incluye como elementos parcialmente integrativos a todos los métodos que existen. En mi exposición boceto desde los primeros renglones la unidad existente en problema y método, según la perspectiva inherente a cada postura, cuestión que no por evidente ha dejado de omitirse en numerosas doctrinas, con pernicioso resultado: separar ambas modalidades del saber cuya realidad adquiere inconmutable correlación; genéranse posturas igualmente nocivas, como son el falso problema que no encuentra un método autónomo para resolverse y el no menos espúreo método que carece de una problemática en la cual verificarse. Al insistir en tal correlación se refuerza la dialéctica epistemológica, que no por conocida ha sido siempre debidamente interpretada.

* Editorial Patria; $1^{*}$ edición, 1960. 
De análoga manera concibo la unidad entre método y fines de la investigación, encarnados en la hipótesis funcional de los valores epistémicos que encuentran progresiva unidad en el paulatino develamiento de la verdad. Creo que no es muy frecuente esta vinculación y su menoscabo redunda en grave perjuicio para la adecuada comprensión del método.

Al concebir dinámicamente la metodología epistémica señalo su indeclinable desempeño formal, que se traduce en la triple proyección del pensamiento, a saber: qué, cómo y para qué pensamos, cuestiones capitales en esta disciplina. Intento asimismo fijar las categorías fundamentales del método, distinguiendo las siguientes: autonomía, objetividad, unidad, totalidad, continuidad y progresividad, cuyo significado explicito debidamente.

He procurado resaltar el nivel jerárquico que adquiere el método a través de los diferentes grados cognoscitivos, desde la metodología universalmente empleada por el conocimiento, hasta los métodos que se aplican a casos concretos de la pesquisa; subrayo la infusión total del método en el conocimiento y la permanencia que exhibe en todas las etapas cognoscitivas, actuando en dinámico paralelismo a la evolutividad dialéctica del saber.

El cuarto capítulo sirve para verificar en el terreno específico las deducciones genéricas cstablecidas en el anterior, asimilando numerosos conceptos que habitualmente son separados de manera abstractiva, pero cuya implicación metodológica resulta incuestionable. Tal es el caso del correlato abstracción-concreción que integra unidad dialéctica por cuya virtud el saber recorre inexorablemente el doble tránsito que supone ir de lo concreto a lo abstracto y de lo abstracto a lo concreto, viaje de ida y vuelta que gira alrededor de la dualidad polar cuyas manifestaciones no constituyen dos métodos, sino uno y el mismo en dos momentos manifiestos.

Análogamente, el distingo que suele efectuarse entre ciencias empíricas y ciencias puras, queda de relieve en su indeclinable unidad como un solo sistema epistémico por el cual las ciencias puras constituyen la racionalización de la experiencia, mientras las ciencias concretas representan la empiricidad de la razón. La dualidad tradicional de los métodos inductivo y deductivo queda de relieve con la unitariedad inherente a un método inductivo-deductivo, al cual se aplican invariablemente las consideraciones anteriores.

En el conocimiento de la realidad reconozco cinco fases transitivas: observación, experimentación, interpretación, formulación y verificación, señalando una secuencia de lo concreto-múltiple a lo normativo-unitario, mientras las ciencias racionales exhiben un método cuyas fases son las siguientes: postulación de axiomas, obtención de teoremas y deducción de conclusiones.

El desenlace obligado de esta excogitación metódica se encuentra obviamente en la dialéctica, que no es sólo dinámica del pensar sino precisamente dialéctica entre la dinámica sintética y la estática analítica, cuyas implicaciones traducen la dualidad existente de hecho en la epistemología material, por una parte, y la lógica formal, por la otra. La dialéctica encuentra su proyección efectiva en la his- 
toria del pensar y particularmente en la historia de la filosofía. De ahí que el tema postrero de este capítulo se refiera al método filosófico, que no se sustrae a ninguna de las características anotadas, sino al contrario, las supone en su más alto grado de unidad y trascendencia. Establezco la correlación dialéctica entre filosofía y ciencia, exhibiendo las categorías correspondientes, aunque debo agregar la misma correlación entre el filosofar y las diversas manifestaciones de la cultura, cada una con su valor privativo.

La acepción de la filosofía como método deriva necesariamente de la postura que sustento, prosiguiendo los lineamientos generales del criticismo, verificable en las categorías del método como crítico, funcional, trascendental, dialéctico, axiológico y filosófico. Finalmente, me ocupa la refutación del individualismo y el irracionalismo en general, no sin apuntar que toda manifestación epistémica, por muy abstracta que sea, tiene su origen primario en un acto vivencial, que a su vez emana de la ignota y connativa potencia del espíritu.

El último capítulo se refiere a la teoría de las ciencias. Parto de sus principios constitutivos y presento una nueva clasificación de las ciencias, enfatizando el carácter apriorístico de las ciencias puras y la modélica síntesis epistémica que es básica para comprender la esencia del conocimiento. Termino este libro con la confronta de la realidad y lo que predica el conocimiento. De esta suerte, Principios de epistemologia es un trabajo que retoma la problemática de la epistemología crítica para aplicar desarrollos adicionales, sin pretender revolucionarla.

En cambio, una apreciable contribución al tratadismo de la materia se encuentra en Principios de estética, ${ }^{*}$ volumen V del decálogo que lleva el rubro genérico de Principios. Su carácter inquisitivo tiene un fuerte matiz heurístico que espero desempeñará importante función para comprender el sentido dialéctico de la estética, habida cuenta del criterio objetivo y dinámico que en dicha obra se establece, así como el desarrollo a que da origen.

Creo que hasta ahora la problemática estética se ha afocado sustancialmente desde dos posiciones disímbolas, como son la llamada "estética desde arriba", que equivale a estética ideológica o filosófica, y la estética empírica o historiográfica, que por análogas razones se denomina "estética desde abajo". La primera corresponde a la asunción ideatoria cuyo catálogo registra por excelencia la filosofía, mientras la segunda se nutre en la toma de noticias que admite esporádicamente la crítica y recoge hilativamente la historia; aquélla es de índole teorética, abstractiva o especulativa, en tanto que ésta exhibe los caracteres opuestos: concretividad, facticidad, singularidad. Dualismo tal de connotaciones refrenda la polaridad categorial que desde siempre ha existido en virtud de las posturas que corresponden a la dialéctica epistemológica; en la oposición de las ideas frente a la realidad se vierte la inveterada dualidad de idealismo y realismo, cuyas vertientes estéticas son bastante características para reconocer en ellas sendas mo-

* Editorial Patria, $1^{9}$ edición, 1958; 3ª 1967. 
dalidades de la inquisición: idealismo o estética desde arriba, y realismo o estética desde abajo.

Postular frente a esta dualidad esquemática la necesidad de una síntesis dialéctica, parecerá normal a cualquier iniciado en filosofía; pero la cuestión no es limitarse a dicha postulación, sino llevarla a efecto mediante la integración dialéctica que requieren las consecuencias sistemáticas. El hecho es que tales consecuencias no son comunes, en el tratadismo estético, donde siguen predominando las disímbolas escuelas precitadas; así se observan la gran mayoría de las doctrinas sobre el arte que informan el contexto de la estética, ya pertenecientes al campo de las teorías lucubrativas, o por el contrario, de los señalamientos empíricos, pecando en el primer caso de abstracción y esquematismo, mientras el segundo adolece casi siempre de la excesiva particularidad a que le circunscribe su desempeño casuístico.

Lo que yo he pretendido en Principios de estética, y creo haber logrado en apreciable medida, es un replanteamiento sistemático del temario correspondiente a la disciplina, en forma tal que recoja las virtudes de los sistemas anteriores sin asimilar sus defectos, cual corresponde a la asunción dialéctico-sincrética de la filosofía moderna. Se trata de una estética apoyada en la base realista que significa el reconocimiento del arte como punto de partida para ìa investigación y la nece: saria gnosis en el plano de las teorías generales que le confiere el perfil doctrinario, crítico, valorativo y filosófico propiamente dicho.

La estética que presento en este marco de condiciones primarias es una estética axiológica, a tono con la equivalencia de cultura y valor que encuentro en las otras disciplinas filosóficas. Ya he dicho que la postulación básica de mi sistema corresponde a la axiomática trascendental, característica también de la filosofía de los valores o axiología; la susodicha equivalencia se observa en que, así como la lógica es axiología de la verdad y la ética axiología de la bondad, la estética se manifiesta como axiología de la belleza, con lo cual hacer estética equivale a emitir un juicio valorativo sobre las obras de arte.

Perspectiva tal, que parece evidente de suyo, no lo es tanto al momento de construir la disciplina; no lo ha sido, cuando menos, la estética ideológica, apoyada casi siempre en opiniones que derivan de algún sistema axiomático admitido apriorísticamente en términos de gusto personal; recae en el consabido subjetivismo que admiten incluso algunos grandes filósofos cuyo pensamiento en otras ramas del saber se sujeta, no obstante, a la objetividad. Sigue pareciendo algo natural que el juicio en torno a la belleza dependa estrictamente de la sensibilidad, desembocando en el consenso subjetivista que afirma al juicio estético como cuestión de gusto, y al gusto como factor constitutivo de los individuos.

Contrariamente a esta proyección subjetivista que predomina aun en nuestros días, principalmente en las teorías filosóficas de la "estética desde arriba", puede y debe asumir la virtud de objetividad, y aún más, de cientificidad, que le corresponde en cuanto disciplina filosófica. He querido bocetar en este libro precisa- 
mente el camino para llegar a la cientificidad del discurso estético, señalando la función que desempeña el arte en cuanto obra producida y acudiendo como criterio postulatorio al principio que ya se ha expuesto del arte como expresión. No descubro ciertamente la tesis precitada, pero la llevo a una verificación cuyas implicaciones casuísticas no encuentran ningún antecedente directo en toda la historia de la estética.

Para mencionar algunas aportaciones que figuran en el libro, enfatizaré la distinción entre estética y ciencias del arte, correspondiendo al paralelo distingo entre la expresión obtenida por la obra y los factores circunstanciales que contribuyen a su realización; dado el predominante influjo que ejercen doctrinas heterónomas como el psicologismo, el sociologismo y el historicismo, dirigir la atención al problema netamente estético en cuanto juicio valorativo de la obra de arte, equivale a innovar las escuelas más conspicuas de la estética contemporánea.

La resolución básica que encuentro para el problema radica en una ecuación fundada entre el contenido, el material y la forma de las obras, atendiendo a los coeficientes indispensables a la creación artística, y por ende, los que participan en todas sus obras. Entiendo por contenido lo expresado en la obra; por material, con qué lo expresa; la forma equivale a cómo lo expresa. Despejo analíticamente la tesis tantas veces esgrimida del arte como expresión, mas no resuelta con igual frecuencia por conducto de las teorias en que se ampara, casi siempre incurrentes en especulaciones "desde arriba", o lo que equivale, producidas al margen de la realidad artística. Contrariamente a la abstracción que de ordinario entraña la estética filosófica, desenvuelvo mi sistema apegado estrictamente a la realidad de las obras donde se define la problemática del arte.

Según este criterio, prosigo la inquisición apoyado en la base expuesta, o sea el concepto del arte como expresión, tendiendo a verificar en todas sus consecuencias la relación de contenido, material y forma, que es la clave de la potencia estética expresiva, la cual equivale a belleza y valor de las obras, o sea el problema privativo de la disciplina.

Partiendo de la ecuación contenido-material-forma, el desenvolvimiento casuístico de la estética tenderá fundamentalmente a verificar el acoplamiento que realizan los tres grandes factores en la esfera del arte, cuya virtud de congruencia y unidad estructural arroja la belleza finalmente traducida en la forma de la obra, subsumiendo al contenido y al material en los términos formales que se observan a cada paso de la creación artística.

En el resto del trabajo - estética especial- examino cuáles son las formas de verificar la ecuación estético-expresiva en las artes particulares y llego a la conclusión que por debajo de la diversificada apariencia en que se manifiestan las obras, subsiste en cada caso la referida función expresiva y comunicativa del arte, ya se trate de un poema, un ensayo o una novela en el sector de la literatura; dibujo, pintura o escultura, si de artes plásticas se trata; una sonata, una sinfonía o un concierto, en caso de la música. La misma relación de contenido-material- 
forma verifícase en artes mixtas como la danza, el canto, la ópera, el cinematógrafo, etc., donde se comprueba desde el primer examen la susodicha función expresiva.

El punto culminante de mis reflexiones consiste en la exploración analítica donde se pone de relieve la adecuación del material a la forma, mediante las reglas técnicas que sirven para organizar la creación artística, y en la contrapartida sintética que destaca la vinculación de las fases constitutivas del arte al servicio de la función común que es la belleza, entendida genéricamente como expresión de los sentimientos. Concluyo en la identidad de técnica y estética, poco admitida en el contexto de las teorías filosóficas y soslayada casi inexcepcionalmente al ubicar los principios generales de esta disciplina, pero básicamente postulada en la preceptiva específica de las artes, como basamento de la actividad práctica profesional.

Con el número 6 de la Serie que estoy comentando se cubre la tarea de las disciplinas fundamentales en el sistema de la filosofía - siempre de acuerdo a la postura que sustento- $\mathrm{y}$ corresponde a la vertiente subjetiva, considerando el coeficiente elevado de subjetividad que se manifiesta en la ética, así como la es. trecha relación que mantiene con los temas que me ocupan posteriormente, o sean la psicología, la pedagogía y la axiología - volúmenes 7,8 y 9 de esta serieasí como la antropología, segundo tomo del sistema. Principios de ética ${ }^{*}$ es una obra que contiene algunas aportaciones de importancia, principalmente en el in. tento de establecer el sistema de la ética sobre las bases que expondré a con. tinuación.

De la obligada necesidad es ubicar a la disciplina como filosofía de la conducta, doctrina axiológica y normativa del deber ser, perteneciente, como las otras, al sistema cultural, de donde el cuadro sinóptico presentado en el primer capítulo, al hablar de la filosofía y el espíritu.

Para situar el fundamento epistemológico de la moral, o sea el conocimiento del deber, exploro las relaciones entre lógica y ética; comento la forma que asume la predicación moral de acuerdo a los cánones de la lógica para exponer finalmente en esta disciplina los caracteres de metodicidad, dialecticidad y cientificidad. También me preocupa la ubicación de la ética en el campo general de la cultura, su extracción humanista, sus relaciones con la religión, con la psicología, la actividad profesional y cotidiana; en suma, con la vida misma.

Especial atención otorgo a las relaciones de la ética con las ciencias sociales, cuestión indispensable para definir el carácter científico de la moralidad frente a las ciencias y las formas culturales que se conectan con ella; tal vez la interfieran e incluso en ocasiones le disputan francamente su problemática; mas a pesar de ello $\longrightarrow$ precisamente por ello- se complementa con las ciencias del hombre, con las otras ramas de la filosofía y todo el saber humano en general.

* Editorial Patria, $1^{7}$ edición, 1960; 37, 1968. 
Destaco las principales, tenidas como ciencias del hombre: antropología, sociolo. gía, economía, política, derecho, historia, psicología, pedagogía y religión. Por último, en esta misma parte de la obra expongo cuáles son las direcciones básicas de la moralidad y las principales corrientes que figuran en la historia de la ética, señalando el papel que desempeña cada una y su mutua conjunción en la unidad sincrética del sistema.

En la segunda parte del libro analizo el sistema de la moralidad en el dominio de la praxis, cuyo denominador fáctico y axiológico está integrado por el acto moral; efectúo en el primer capítulo un pormenorizado análisis de dicho acto, señalando la participación de la conciencia, sus elementos integrativos, la calificación y los valores, fines y medios, la idea de lo bueno y la postulación o electividad moral. El sistema de la ética se lleva a cabo mediante el conocimiento del acto moral, de los valores y lo humano, en el seno de una convivencia situada bajo el potente influjo de las costumbres y tradiciones, a las que opone el individuo la responsabilidad del deber mediante su convicción en la buena voluntad, así como el fomento de las virtudes por medio de la educación.

Este enfoque integrativo adquiere peculiar dinámica al hablar de la dialéctica moral, estableciendo la correlación de las diversas doctrinas éticas y las nociones de ser, deber, libertad, individualismo y colectivismo, etc., señalando el paulatino e ilimitado incremento del territorio normativo frente al electivo. El análisis de las virtudes resulta obligado en todo sistema moral; a la clasificación aristotélica de las virtudes éticas y dianoéticas, agrego las virtudes prácticas, que en conjunto presentan los elementos constitutivos del carácter formado en la educación moral. Por último, el distingo entre ética autónoma y ética heterónoma sirve para contrastar la posición libertaria del individuo que se gobierna a sí mismo, frente a las actividades que requieren el sacrificio de la libertad individual o lo prefieren sobre su conservación.

El libro finaliza con una exposición sucinta de las doctrinas morales, en las que distingo fundamentalmente cinco grandes direcciones, correspondiendo a la ética empírica, la idealista, relativista, formalista y material, respectivamente. La orientación predominante en este capítulo tiende a situar a cada una de tales corrientes como representativa de un momento significado en la metódica de la moralidad, pues la elección del sistema ético requiere un basamento en la realidad, que proporciona la ética empirica, pero al mismo tiempo reclama la ascensión unitaria a la idea moral que se encuentra en la ética idealista; como el valor realizado en todo sistema es siempre relativo, de ahí la urgencia de una ética relativista; por su parte, la necesidad formal de acatar el mandato normativo se encuentra en la ética formal, que contrasta con la influencia de los sentimientos subjetivos cuya acción pragmática ha sido expuesta en la ética material.

A partir de la fundamentación sincrética de las doctrinas morales, efectúo una brevísima exposición de sus problemas básicos, tendiendo a destacar el aporte que cada una proporciona al sistema conjunto de la ética y la moralidad, situado 
dialécticamente en la dinámica metodológica, por cuya virtud adquiere pleno significado cada una de sus doctrinas constitutivas. El último capítul del-libro se refiere al problema de la acción moral, integrado por la infinidad de actos concretos que se registran en la vida cotidiana, cada uno de los cuales debe ir fundado y regulado por un criterio ético, que a su vez se apoya en la apreciación integral donde se arbitra una multiplicidad de criterios aplicables dialécticamente al enjuiciamiento casuístico de la acción moral.

El último de los libros que hasta ahora he terminado para la Serie es Principios de psicologí," con el número 7 en el orden de la misma y cuya naturaleza boceté anteriormente a grandes rasgos. La postura que sostengo frente al problema psicológico tiene como postulado que, por una parte, se independiza materialmente de la filosofía, aunque por otra mantiene un doble nexo formal con la misma, en cuya virtud incluyo este libro en el sistema que es básicamente filosófico. El primer nexo entre ambas ciencias radica en un tratamiento que podría llamarse la perspectiva filosófica de la psicología, entendida en el sentido metodológico, de análoga manera a como lo hice en el caso de la antropología. Esto quiere decir que mi propósito tiende fundameniahimentc a una revisiońn, revaloración y sistematización de los conceptos psicológicos, bastantes de los cuales muy confusos se encuentran en el campo de la propia disciplina, incluyendo algunos de importancia capital.

El hecho de no pertenecer profesionalmente a la psicología, aunque enterado de ella, puede representar una considerable ventaja respecto a la postura normalmente asumida por el psicólogo profesional, casi siempre especialista y que por consecuencia observa única o preferentemente la problemática que encuentra en su dominio, produciendo marcada incomprensión frente a otro género de doctrinas. Baste señalar, a título de ejemplo, el duelo emprendido entre los partidarios de la escuela biologista y los devotos del espiritualismo, para percatarse del estado imperante en ese problema. Además, la proliferación de escuelas psicológicas obliga a promover una sistematización de las mismas, que abordo en cuatro grandes sectores, correspondientes a la psicología básica, la psicología biológica, la psicología analítica y la psicología social, cuya clasificación puede ser de gran importancia para captar el panorama conjunto de esta disciplina y estimar adecuadamente cada una de sus derivaciones particulares.

La psicología misma, en cuanto estudio de la manifestación interconsciente del ser humano, reclama una amplia participación del conocimiento filosófico y genéricamente cultural, a través de su conexión directa con la antropología, la pedagogía y la axiología, que a su vez se proyectan en el amplio mundo de actividades y conceptos relativos al ser humano. Me parece que sobre esta perspectiva general he logrado algunas apreciaciones originales.

El punto de vista sincrético que sustento en relación a la psicología es el mismo

* Editorial Patria, $1^{4}$ edición, 1969. 
que he esgrimido frente a otras disciplinas; consiste en estimar la diversidad de posturas que aparece en las mismas, como resultado de una diversidad posicional, estudiando en conjunto una problemática que en el fondo resulta común a todas ellas, si bien cada una la afoca con su criterio privativo, a su vez derivado del problema que le corresponde tratar en la heterofolia que resulta por la compleja índole del problema en cuestión. El efecto de aplicar semejante criterio vinculatorio conduce a una especie de eclecticismo, que mejor llamo sincretismo, por el cual cada una de las posturas filosóficas y psicológicas tiene su razón de ser, misma que puede justificarse siempre y cuando se observe el problema respectivo como lo que es, o sea un problema específico relacionado con una multiplicidad de criterios que derivan de otros problemas, y a su vez corresponden a sendas manifestaciones de una misma realidad, que es en este caso el psiquismo, o la conducta del ser humano.

La primera de las grandes corrientes psicológicas que puede reconocerse históricamente, corresponde a la psicología de tipo filosófico, y en cierto modo religioso; representa el estado antelativo a la obtención de una psicologia científica, con la especial importancia de contener embrionariamente las doctrinas filosóficas que observamos en reflexiones ulteriores. Empero, éste no es más que un antecedente para llegar a la verdadera psicología, la realización autónoma y sincrética de dicha ciencia; autónoma, porque delimita adecuadamente sus tareas y métodos propios, mientras sincrética, porque incluye todas y cada una de sus direcciones, a las cuales corresponde un basamento común en los fundamentos de la disciplina. En términos generales la considero como psicología básica, concepto que corresponde en parte a la antigua psicología descriptiva y representa el primer estadio integrativo en la erección autónoma de la misma.

Una de las manifestaciones particulares en la psicologia corresponde a la que exhibe orientación biológica, debido a la definitiva influencia que ejercen los factores biológicos en la conducta; esta dirección se ha constituido principalmente a partir del siglo pasado, con el perfeccionamiento de las ciencias experimentales, entre las que se cuentan la física, la química y obviamente la biología, cuya influencia ha sido de primer orden para la explicación objetiva del comportamiento, según las condiciones biofísicas que imperan en el mismo. De ahí estudios tan importantes para la fisiologia conductista como la endocrinologia, la neurología, la bioquímica y demás, que arrojan poderosas luces a la explicación del psiquismo, aunque de ningún modo lo abarcan en su totalidad, como desearían los partidarios radicales de esta corriente. $\mathrm{El}$ problema que me preocupa deslindar al respecto es la circunscripción que señala el verdadero alcance y las limitaciones de la orientación biológica en psicología.

Un tercer capítulo está integrado por la psicología analítica que corresponde a la escuela iniciada por Freud y proseguida en multitud de trayectorias diferenciales que mantienen lo fundamental del método psicoanalítico, aplicando criterios diferentes y complementarios en los cuales se integra la perspectiva con- 
junta de la propia disciplina. El punto de vista que esgrimo en la exposición tiende a poner de relieve la complementariedad de dichas posturas y, por consecuencia, la esterilidad del injustificable antagonismo en que suelen manifestarse. De manera especial he querido contribuir al esclarecimiento de los temas psicoanalíticos a la luz de su inherente unidad metodológica y sistemática.

La última parte del libro está dedicada a la psicología social, que logra la más completa polarización de las doctrinas psicológicas, que se manifiesta en forma colectiva y traduce la dinámica interhumana del psiquismo. Entre varias otras apreciaciones de carácter constitutivo quiero señalar el distingo que llevo a cabo entre psicología de sociedades, de masas, y de multitudes, cuya diferencia suele quedar inadvertida en el tratamiento de tan complejo problema; al mismo tiempo, empleo ese tratamiento para vincular el desarrollo de la psicología al esquema general de la cultura, considerada ésta como producto culminante de la actividad social. El interés que encuentro al situar el estudio psicológico en un cuadro dedicado a la filosofía de la cultura, estriba precisamente en que la esencia de esta última no puede ser comprendida sin referirla al origen subjetivo, situada en la dinámica intrapersonal en que se originó; de ahí proviene no solamente la correlación de psicología y filosofia, sino también de ambas con respecto a la pedagogía, que ocupa el siguiente volumen de la serie.

Tal es, pues, el diseño que me sirve de base para desarrollar el problema de la psicología, según los lineamientos expuestos. Me parece que las aportaciones contenidas en este libro convergen a la común preocupación de establecer la unidad sincrética de la disciplina, hoy por hoy cuestión la más urgente de una temática que, en los demás aspectos, registra un portentoso avance, principalmente en el sector experimental, sobre el que encamina sus pasos, cada vez más amplios, en su desarrollo como ciencia autónoma, aunque no siempre lleva aparejada la cabal conciencia metodológica y sincrética que reclama la acelerada complejidad de la psicología.

He querido presentar un juicio general sobre los siete libros que hasta ahora he publicado en la Colección "Principios", de la cual ya informé que dista mucho de ser un conjunto de obras aisladas ni divulgatorias; por el contrario, en esta serie intento poner las bases - y creo lograrlo en apreciable medida- para la construcción del primer sistema filosófico que se expone y realiza en lengua castellana, cuando menos en la época moderna.

Ese intento está señalado básicamente desde el primer libro que publiqué, intitulado Reflexiones en torno a la filosofía de la cultura." Este libro es una investigación que tiene cierta importancia no sólo por contener rendimientos inquisitivos que me encargaré de bocetar a continuación, sino también porque el ejercicio sistemático viene siendo tan exótico en nuestro tiempo y en nuestro ambiente, que inclusive conspicuos pensadores dudan de que pueda ser una vía adecuada para

*Colección "Cultura Mexicana". U.N.A.M., 1" edición, 1956. Agotado. 
filosofar, a tono con el sentido esporádico de un ensayismo rapsódico, que se ha puesto de moda en la mayoría de los ambientes profesionales, seguramente como compensación, pseudoteorética, cuando escasean los tratados sistemáticos de envergadura. Por lo que a este libro respecta, se pueden aplicar las consideraciones que expuse anteriormente en relación al proyecto de formular un sistema basado en la equivalencia axiológica de las disciplinas filosóficas, con lo cual se trasciende el tono generalmente asistemático que se encuentra en la filosofía de lengua castellana. Claro es que el contenido del libro resulta elemental frente a la pretensión de sistema; apenas se le podrá tener como índice de varios trabajos a desarrollar, lo cual desde luego no es óbice para que en sus propios alcances y con las señaladas limitaciones, produzca una aportación inquisitiva.

Así, en el capítulo primero expongo el concepto que reviste la filosofía de la cultura a través de su desarrollo occidental; la interinfluencia de filosofía y cultura en el encuadre de sus coordenadas históricas según la sensibilidad de Occidente; para ello acudo a la clasificación tradicional en ocho periodos históricos, destacando el carácter de cada uno y la influencia que en todo caso ejerce la cultura en el pensamiento filosófico. La conciencia histórica del filosofar conduce a una historia de la filosofía, así como una historia de la historia, una historia de la filosofía de la historia y una filosofía de la historia de la filosofía, elementos todos que determinan una constelación funcional donde se manifiesta el proceso integrativo del filosofar cultural en la dimensión histórica y sistemática, diacrónica y sincrónica, o, para decirlo en una palabra, diasincrónica, que es el verdadero concepto sincrético y unitario de la constitución intelectiva.

Algo similar ocurre con la filosofía y el método; en cada postura o problema se encuentra la respectiva dimensión metodológica que desempeña una función determinada; por ello, así como puede hablarse de la integratividad histórica y sistemática, también cabe investigar el criterio metodológico integrativo. Señalo que la condición metódica para filosofar es la libertad de conciencia, a la cual acompaña la conciencia de la libertad. En virtud de la libertad se justifica el progreso en la cultura y mediante la conciencia de la libertad en el filosofar, se erige la autoconciencia individual y colectiva del hombre libre.

Establezco una distinción entre los conceptos de cultura propiamente dicha, que es conciencia objetiva, y metacultura, que equivale a autoconciencia, así como un primer concepto genético que denomino paracultura, o sea la cultura surgiente al plano objetivo y autónomo, que registra inevitablemente una decisiva influencia de la heteronomía derivada de los distintos factores colaterales y contributivos al hecho cultural.

La culminación del método es la ley regulativa que justifica y exige el camino infinito de elaboracicón progresiva, partiendo de la libre electividad que adopta. al siguiente como axioma ejercitivo: la razón del espíritu es la libertad y la razón de la libertad es la ley. Entiendo al concepto de ley, genéricamente como valor, de suerte que el valor de cada especie indica el tipo de ley imperante en cada forma 
de actividad cultural; valor no es sólo finalidad axiológica o electiva, sino tam. bién estructura formal que condiciona su realización mediante el criterio objetivo de la verificabilidad concreta.

De ahí opino que sólo existen tres disciplinas filosóficas fundamentales: lógica (y/o epistemología); ética y estética, acompañadas por una unidad esencial que denomino propedéutica, integradas diacrónicamente a través de la histórica con un basamento común que es el método, lo cual, sumado a la acepción general de axiología, incorpora también a las ciencias de interés centralmente subjetivo, como la psicología y la pedagogía, que en cierto modo complementan la perspectiva integral del sistema filosófico.

Rechazo la posibilidad de una filosofía intuicionista, y también de la metafísica, si se entiende como conocimiento directo, abstracto y universal del ser; por último, disciplinas tales como la filosofía de la religión y del lenguaje, quedan en calidad de historia y metodología de las ciencias respectivas, o sea como disciplinas formales que por sí no arrojan conocimientos empíricos sino organizan y fundamentan una gama de ciencias concretas que de ello se ocupan.

Ahora bien, los conceptos dinámicos del filosofar se correlacionan a partir de la aceptación de ios valores como basamento indispensabì para justificar aì pensamiento filosófico y la cultura en general; a su vez, el sistema filosofía-cultura-valores, se funda en la noción del progreso, pues solamente progresa lo que vale y solamente vale lo que progresa. Por otro lado, progreso y valor traducen su objetividad en acontecimientos realizados, sin olvidar el origen subjetivo que poseen inexcepcionalmente, de donde la necesidad de acudir también al estudio psicológico y pedagógico de los hechos para lograr una explicación integral de la cultura. En tal aspecto, las doctrinas subjetivistas e irracionalistas tienen cabida como contrapolo genético del teorético que equivale a objetividad y razón; de ahí que a las impresiones individuales no se les pueda conceder un estricto valor objetivo. Sostengo la tesis de que la única filosofía posible es la filosofía de la cultura; los demás sistemas, o bien se reducen a un momento integrativo de aquélla, o por el contrario, se encuentran fuera del encuadre cultural y no podrán demostrar plena validez objetiva.

El segundo capítulo del libro, relativo a la Lógica, toma como punto de partida la noción que expuse antes: unificar lógica y epistemología en cuanto disciplinas del conocimiento, con la única posible diferencia de que la lógica podría entenderseprincipalmente en la acostumbrada acepción formal y analítica, mientras la epistemología viene a ser equivalente a doctrina dialéctica y material del pensamiento. Asi la entiendo y por ello el capítulo respectivo contiene observaciones diversas posteriormente contenidas en Principios de epistemología, dejando a la lógica propiamente dicha los temas formales que, por ello mismo, se encuentran directamente vinculados a la tradición escolástica.

En esta inteligencia aplico el concepto de lógica como doctrina del logos, que a su vez equivale lato sensu a razón explicativa, fundamentación teorética o re-- 
flexión metodológica, concepto que se ha postergado un tanto en el pensamiento contemporáneo para ceder prioridad a la moderna lógica formalista de corte matemático.

Directamente aparejada al logos se encuentra la noción de autonomía: el logos mismo afocado a la autorrealización de cada disciplina en función de su valor privativo, de donde autonomía es tanto como propiedad intrínseca y objetiva del valor, para distinguirla de los factores genéticos que corresponden a las ciencias del pensar. Éste es uno de los principios fundamentales que extiendo a todo el campo cultural en el sistema filosófico-crítico, cuya objetividad considera en un primer plano de importancia a la realidad objetiva y funcional del valor.

También entiendo a la lógica-epistemología-axiología en calidad de una metódica fundamental de la cultura, originada en la función axiológica que distingue a cada una de sus modalidades. Por ello defino a la lógica como autoconciencia del pensamiento en función objetivadora, y se distingue de las ciencias particulares que se ocupan de estudiar al conocimiento desde un ángulo distinto, cual corresponde a los factores concretos del pensar -psicológico, sociológico, histórico, etc.cuanto de la metafisica, pretendida ciencia que, siguiendo la tradición kantiana, considero infundada e incapaz de verificar objetivamente sus aseveraciones, en cuyo caso dejaría de ser metafísica para convertirse en ciencia. La mejor de sus posibilidades, como antecedente de la ciencia particular, si pretende adquirir función explicativa y concreta, vale científica, corre el riesgo de convertirse en repetidora de la ciencia particular. La tesis idealista afirma que la realidad es asequible sólo a través del pensamiento y por ende carece de sentido hablar de una "realidad en si", tesis que - no es necesario decirlo- se debe a la paternidad de Kant y, más remotamente, de Platón. Efectúo un breve repaso de algunas posturas importantes en la historia de la lógica para mostrar cómo a través de ella se integra paulatinamente la unidad del proceso epistemológico, y por consecuencia, el objeto de la lógica.

Ahora bien, el nódulo de las consideraciones lógico-epistemológico-axiológico-metodológicas se encuentra en la síntesis dialéctica del conocimiento que presenta la base para el desenvolvimiento de esta disciplina; efectúo una reexposición de la tesis elaborada parcialmente por Kant, Fichte, Hegel y Croce, con un sentido distinto al que se encuentra en cada uno, evitando el abstractismo kantiano, el formalismo fichteano, el metafisicismo hegeliano y el romanticismo croceano, aunque de cada uno somos todos necesariamente tributarios en la medida que no es posible prescindir de la categorética forjada por Kant, el cientificismo funcional de Fichte, la dialéctica histórica de Hegel y el sincretismo actualista de Croce.

En este intento de integratividad sintética radica la probable aportación que ofrezco en el capítulo. Otro tanto sucede con el problema de la verdad, que asimilo al concepto funcional de la realidad, cuya esquematización logística se encuentra en la matemática. En cuanto a los principios lógicos supremos, promuevo zuna crítica al enunciado tradicional, su sentido formalista y esquemático, el cual 
implica en cada caso un correlativo axioma dialéctico que sostiene lo contrario del principio correspondiente en la lógica tradicional. Por otra parte, la concepción de las categorias lógicas se inspira originalmente en la metafísica aristotélica y con mayor ductilidad en la Tabla kantiana, primer intento registrado en la historia para establecer el sentido dialéctico de las categorías.

La declarada filiación criticista debió conducir forzosamente a una severa crítica de la metafísica, que considero — siguiendo básicamente a Kant- simple y sencillamente como inevitable error histórico, producido por la precipitación del hombre al querer determinar lo que se encuentra todavía fuera de su alcance científico; o bien radica en la confusión de su temario con otras ciencias, en cuyo caso no hace más que duplicarlas. Sostengo, en conclusión, que la metafísica, gozando aún de bastantes adeptos, es incapaz de proporcionar un solo conocimiento objetivamente válido que no corresponda a ninguna de las otras disciplinas filosóficas y no se encuentre como problema planteado en alguna de las ciencias particulares que existen.

El capítulo de la ética principia con un señalamiento general de su historia, según el criterio empleado en su erección autónoma, aunque paralelamente y en rontraste regístranse las desviaciones heterónomas que hacen depender a la conducta de factores distintos al libre y consciente ejercicio de la voluniac. Púsiulo el concepto de una historia ideal, o sea la historia de la ética autónoma, frente a una historia real, que incluye todas suertes de manifestaciones y doctrinas relativas a la conducta. La historia ideal de la ética es historia de la libertad, de la igualdad y demás conceptos que concurren a determinar la autonomía moral con base en la equidad humana.

Por otra parte, distingo en ella dos grandes territorios, como son la ética pura y la ética concreta; aglutina la primera al conjunto doctrinario que representa la idealidad moral, de acuerdo a los valores progresivos postulados por el espíritu, básicamente la afirmación consciente y normativa de la libertad, teniendo en cuenta la restricción que impone el imperativo ético. Derivan sus relaciones a la sociología, la política, la pedagogía, la antropología, la religión; en suma, a las ciencias del hombre.

En este capitulo presento las interrelaciones de la ética y las ciencias sociales, que extendería ulteriormente en los Principios de ética. Por su parte, aplico la tesis de que la ética concreta tiene por objeto establecer la doctrina correspondiente a la realización moral en el seno de la praxis, cuya base radica en la teoría de las virtudes como potencias o capacidades de acción. De manera especial señalo en qué consiste la dialéctica de la moralidad, como correlación de lo exigido, lo prohibido y lo permitido por la ley moral; destaco las profundas vinculaciones con el derecho, la historia y la sociología, en cuyo seno tiene efecto la consumación de la praxis y la justificación problemática de la ética concreta. Llego finalmente al sistema de la ética social, comprobando la mayor penetración de las circunstancias teoréticas y fácticas que actúan en la colectividad, prin- 
cipalmente la concatenación de fines y causas, o sea la comunicación del plano teleológico y el causalista en el seno de la actividad social. Asimismo, el origen ético que se observa en la constitución de las relaciones societarias y comunitarias.

Apunto, por último, el concepto de la sociología como metodología formal de las ciencias sociales, con la facultad de proyectar su tarea concreta a través de las disciplinas básicas que, además de las citadas, son el derecho, la historia, la economía y la pedagogía, concebida esta última en el sentido clásico: educación de la personalidad en la vida y sus valores.

Dentro de esta perspectiva general creo haber desarrollado un tratamiento de la ética que, sin ser revolucionario, tiene cuando menos el mérito de refrendar algunos aspectos casi olvidados en el dominio de la ética criticista, que considero fundamentalmente como una ética relativista, y al mismo tiempo el único sistema operativo de la ética, sujeta como se encuentra a las múltiples condiciones determinantes de la conducta que, por ello mismo, constituyen otros tantos coeficientes de relativización moral.

El aspecto contributivo de este capítulo se localiza principalmente en el acopio de datos estructurales que establece la función del propio relativismo, pues no existe ningún sistema ético que sea concreto y pueda considerarse universalmente aplicable a todas las sociedades, a todos los individuos, a todos los proble. mas y todas las épocas de la moralidad; oscilante es el concepto de lo bueno y lo malo de acuerdo a los factores ambientales y circunstanciales que envuelven a la conducta; dichos factores son de orden físico, económico, sociológico, histórico, psicológico y antropológico, pues en cada uno se contiene un aspecto condicionante del comportamiento. De ahí la necesidad de relativizar los principios de la ética según las condiciones que determinan positivamente a la conducta, en vez de pronunciarse por el utopismo ideológico a que tan dadas son algunas direcciones de la materia.

En el capítulo respectivo realizo un examen conducente al propósito que acabo de expresar, tendiendo a integrar el sistema de la ética en sus diferentes aspectos; me parece que lo esencial es vincular el polo ideal y teorético de la ética pura con el real y pragmático de la ética concreta, examinando en cada caso las relaciones que concurren a la integración del sistema. La contribución ofrecida en este capítulo radica en la imagen integral del problema ético; si bien es probable que la gran mayoria de los puntos concretos hayan sido tratados en el seno de la filosofía moral y las ciencias de la sociedad, no conozco ninguna doctrina, ningún sistema sincrético, en el cual dichos puntos se estructuren como se encuentran en el tratamiento de referencia.

El cuarto capítulo se refiere a la estética, cual fue expuesta desde entonces en calidad de doctrina axiológica del arte, a diferencia de las posturas empiriológicas que se cobijan en la crítica historicista y la estética sociologista, llamada también "desde abajo" y distinguida de la estética filosófica, ideológica o especulativa, que se considera "desde arriba". Contrariamente a estos planteamientos 
predominantes en el comün tratadismo de la disciplina, presenté desde mis primeras exposiciones la necesidad de manejar a la estética con un método que no sea aisladamente "desde arriba" ni "desde abajo", coordinando el derecho que tiene la primera a captar los datos aportados por la experiencia, y la segunda a obtener teorías generales y probablemente universales, que expliquen la normatividad del hecho artístico. Ahora bien, como no es posible lo primero sin lo segundo, y recíprocamente, resulta de ahí la idea fundamental que presento en este capitulo, consistente en la estética dialéctica cuyo método radica en la autonomía, lo cual equivale a reconocer y respetar la estructura axiológica del arte, el más indefectible y auténtico problema de la estética.

El primer enfoque para integrarla sistemáticamente tiende a definir la objetividad del arte como expresión del sentimiento, en lo cual radica el valor obtenido por la obra; la tesis que sostengo está completamente apegada a la realidad del arte, y trasciende el criterio subjetivista empleado cuando se habla de sentimien. tos, para afirmar la forma de realización como estructura de un contenido material que cristaliza en la primera por virtud de su propia constitución emotiva.

El complejo panorama que presentan las diversas artes particulares me llevó a concebir una clasificación de las mismas, basándome en la categoría estética realizada en cada caso: el espacio visual en las artes plásticas, el iiempo auditivo en las artes rítmicas y el símbolo metafórico en las artes literarias. Me parece que es la primera ocasión - cuando menos no conozco otra- en que la categoria esencial de cada arte, y no el material, el contenido o la forma, aisladamente considerados, se aplica a elaborar una clasificación conjunta de las artes.

El postrer apartado de este capítulo se refiere a la vivencia artística; allí establezco cuál es el verdadero sentido que adquiere la subjetividad en la creación y recreación del arte; resulta incuestionable no sólo su amplia participación, sino la gran importancia que asume lo subjetivo en la vivencia artística y que debemos reconocer sin mengua de la importancia intrascendible que corresponde al arte mismo en cuanto realización objetiva de los valores estéticos. Por ello, el enfoque sobre la vivencia artística sirve para evitar la frecuente confusión de lo bello y lo valioso en el arte, con el proceso subjetivo en el cual se origina.

De manera especial quiero mencionar la primera parte de este capítulo, relativa a la Historia de la estética, pues en él planteo por vez primera la concepción de una historia sistemática entendida como doctrina axiológica de la belleza artística y realizada a través del método dialéctico que señalé antes. La originalidad de ese tratamiento radica en que no me limito a señalar las opiniones históricamente producidas, sino que las considero en función del aporte ofrecido a la consecución de la estética axiológica, circunscribiendo en este ámbito a la verdadera historia de la estética, en medio de las numerosas ideas que se han pronunciado en relación al arte, la belleza y los sentimientos, cuyo conjunto constituye la temática tradicional de esta disciplina.

El último capítulo se refiere a la historia y axiología de la cultura. Sitúo diná- 
micamente el problema que figura en el motivo conductor del trabajo, a saber: la génesis dinámica y la evolución de los valores en el seno de la cultura. Principio deslindando el concepto de historia, al distinguirla por una parte como acaecer, y por la otra, como doctrina sobre el acaecer; también la doctrina de las doctrinas del acaecer, que se traduce en la filosofía de la historia, y la historia de la filosofía de la historia, como hay también una historia de la filosofía que conmotiva una filosofía de la historia de la filosofía.

Aprovecho la distinción tradicional de naturaleza y cultura, considerando a la primera como realización causal y a la segunda como teleológica, para refrendar la distinción criticista de ciencias nomotéticas e idiográficas, que corresponden a sendas formas de normatividad. La historia pertenece a este último grupo y se manifiesta como doctrina de lo singular, de donde la importancia del análisis en la categoría de singularidad.

Con parejo fundamento rechazo la idea arcaica de la historia como reseña de los hechos pretéritos, adoptando el sentido integral del historiar; presento como básicos momentos de la historiografía a la ordenación, la reconstrucción y la interpretación de los hechos que integran el acaecer. Tales son las tres fases que me parecen constitutivas del método histórico, el cual debe complementarse con el criterio de valor, pues la facticidad se integra con un número ilimitado de acontecimientos simultáneamente producidos, muchos de los cuales - la gran mayoría, podríamos decir- resultan inesenciales y no tienen por qué figurar en el tratadismo histórico, el cual supone la explicación de los hechos importantes que se presentan en la experiencia de los individuos y los pueblos, reconocida a través de su manifestación cultural. Una historia sin criterio selectivo sería narración caótica de acontecimientos indiscriminados.

De manera especial creo un aporte el brevísimo estudio que llevo a cabo sobre el método de la historia, considerándola no sólo como una ciencia, sino con derecho a ostentar el adjetivo de rigurosa, siempre y cuando se someta a la evolución progresiva del saber, que para mí está contenida en los tres grados de la categoría kantiana de la modalidad: posibilidad, realidad y necesidad. El método histórico se manifiesta inicialmente como señalamiento de los hechos en el nivel de la posibilidad fáctica, de donde procede a la selección y reestructuración ideográ. fica para dirigirse a la realidad empírica y ascender mediante la explicación de sus formas normativas a la necesidad teorética. El reconocimiento de lo apodíctico en la historia es una de las aportaciones que intento ofrecer en esta obra, amparándome en el concepto necesitante de los valores que, en pureza de su concepción ideal, imponen indefectiblemente su normatividad a la evolución casuística de lo real.

Naturalmente, el referido criterio selectivo no permite llegar a otra conclusión que la historia como historia de la cultura, desde el momento que el acaecer experiencial está saturado por una determinabilidad axiológica que establece el sentido regulativo de sus orientaciones valorativas. La integración de la doctrina 
histórica permite afirmar que, en paralelo al distingo establecido en los anteriores capítulos, también existe lato sensu una historia real junto a una historia ideal, siendo la primera al acaecer tal como se produce en la contingencialidad empírica de los hechos, o sea el problema que se presenta a la historiografía, mientras la segunda es historia afocada de preferencia a la realización de los valores, que equivale a explicación y justificación de los hechos constitutivos de la cultura. El estrecho nexo entre filosofía e historia me compele al reconocimiento de que, al mismo tiempo de ser filosofía de la cultura, es una filosofía de la historia de la cultura; si el objeto del cual se ocupa tiene una raíz indeclinablemente histórica, no podrá trascender esta condición, que es al mismo tiempo la mejor garantía para reafirmar la ley que rige su dinámica evolutividad.

Instituto de Investigaciones Filosóficas

Miguel. Bueno

Universidad Nacional Autónoma de México 